\title{
Viscous Fluid Cosmological Model in (2+1) Dimensional Space-Time
}

\author{
G. R. Avachar \\ Department of Mathematics, S.S.E.S. Amt's Science College, R.T.M. Nagpur University, Nagpur, India
}

Available online at: www.isroset.org

Accepted:14/Aug /2018, Online 30/Aug/2018

\begin{abstract}
The viscosity fluid cosmological model in (2+1) dimensional Robertson-Walker space-time, when the source for energy momentum tensor is bulk viscous fluid has been investigated using the special law of variation for Hubble's parameter proposed by Berman and the bar tropic equation of state. The physical and kinematical parameters of the model are discussed. The model is expanding with time since $q+1>0$. The model has no initial singularity at $t=0$. It is observed that, the Hubble parameter $H$, expansion scalar $\theta$, matter density $\rho$ and pressure $p$ decreases with time and approach zero as $t \rightarrow \infty$ and all diverges at $t=0$. Also shear scalar $\sigma$ and anisotropic parameter $A_{m}$ vanishes, which indicates that shape of the universe remains unchanged during the evolution and universe becomes isotropic and shear free. It is observed that the model is expanding, nonsingular and non-rotating. It is also observed that model remains isotropic throughout the evolution and the bulk viscosity in the model decreases with time leading to inflationary model.
\end{abstract}

Keywords-Cosmology, $(2+1)$ dimension spce-time, viscous fluid

\section{INTRODUCTION}

Lower dimensional models have been of enormous use in practically every other branch of physics. Such models are important because they help to generate new ideas, and to stimulate new insights into their higher dimensional counterparts. Moreover, they provide a simple setting in which certain basic physical phenomena can be easily demonstrated, while avoiding the mathematical complexities often encounter in four dimensions. Therein lay the motivation for studying gravity in three space-time dimensions.

$(2+1)$ dimensional gravity does contain interesting features common with four-dimensional gravity. Einstein gravity in three space-time dimensions exhibits some unusual features, which can be deduced from the properties of the Einstein field Equations and the curvature tensor.

Deser, Jackiw and 't Hooft [1] have obtained the solutions to three dimensional Einstein gravity with mass less, spinning point source, and Clement [2] has generalized their results to include many massive spinning sources. The generalization to coupled Einstein-Maxwell theory has been considered by Deser and Mazur [3], Melvin [4] and Gott, Simon and Alpert [5]. The Regge calculus version of 3-D dimensional gravity with point masses has been developed by Rocek and Williams [6]. Many of the basic aspects of classical Einstein gravity in three dimensions are covered in the article by Giddings, Abbott and Kuchar [7] Gott and Alpert [8] and Barrow, Burd and Lancaster [9]. They discussed the lack of correspondence between Einstein and Newtonian gravity in three dimensions; the conic geometry associated with a point mass and also includes cosmological solutions for perfect fluids. In addition, Barrow, Burd and Lancaster present two cosmological solutions containing scalar field that produce inflation, and discussed cosmological singularities for 3-D space-time. Deser and Laurent [10] have studied the interior and exterior solutions to various matter distributions assuming the space-time is axially symmetric and stationary. Deser [11] has shown that there are no nontrivial statics solutions to the coupled Einstein gravity-Yang Mills system in three dimensions. Edward Witten [12] has shown that (2 +1 ) dimensional gravity (with or without a cosmological constant) is exactly soluble at the classical and quantum levels and it is closely related to Yang-Mills theory with purely the Chern-Simons action. N.J. Cornish and N.E. Frankel [13] have investigated gravitational field theories in $(2+1)$ space-time dimensions and reviewed the consequences of the lack of a Newtonian limit to general relativity. The cosmic holographic principle suggested by Fischler and Susskind has been examined in $(2+1)$ dimensional cosmological models by Bin Wang and Elcio Abdalla [14]. Ranjan Sharma et.al [15] have investigated Gravitational collapse of a circularly symmetric star in an $(2+1)$ anti-de-Sitter space-time and analyzed the impacts of various factors on the evolution of the star, which begins its collapse from an initial static configuration. Yun $\mathrm{He}$ and Meng-Sen Ma [16] have constructed $(2+1)$-dimensional regular black holes with nonlinear electrodynamics sources and studied the thermodynamic properties of the regular black holes.

Several authors have investigated cosmological models in different theories of gravitations with viscous fluid as a source. Reddy et al. [17] have investigated Kaluza-Klein 
bulk viscous cosmic string universe in $\mathrm{S}^{\prime}$ aez-Ballester theory [18]. Naidu et al. [19] have discussed the same universe in Brans-Dicke [20] scalar-tensor theory of gravitation. Reddy et al. [21] presented LRS Bianchi type-II universe with cosmic strings and bulk viscosity in the $f(R$, $T$ ) theory of gravity proposed by Harko et al. [22] while Reddy et al. [23] have studied Kaluza-Klein bulk viscous cosmic string model in $f(R, T)$ gravity. Naidu et al. [24] have obtained a Bianchi type-V bulk viscous string model in $(R, T)$ gravity. Reddy et al. [25] have discussed LRS Bianchi type-II bulk viscous cosmic string cosmological model in the scale covariant theory of gravitation formulated by Canuto et al. [26]. D. R. K. Reddy and et.al [27] have investigated Bianchi type- $\mathrm{VI}_{0}$ model in scalar-tensor theory of gravitation proposed by Saez and Ballester [28], when the source for energy momentum tensor is a bulk viscous fluid containing one dimensional cosmic string. Shri Ram and Priyanka Kumari [29] have presented non-singular Bianchi types I and V cosmological models, in the presence of bulk viscous fluid and within the framework of $f(R, T)$ gravity theory. Very recently Mishra R K and Chand A [30] have investigated viscous string Cosmological models in scalartensor Brans-Dicke gravity [31]

Motivated by the above investigation of viscous fluid in various theories of gravitation, we in this paper investigate viscous fluid in $(2+1)$ dimensional gravity theory. The paper is organized as follows. Section II, deal with the derivation of the field equations in $(2+1)$ dimensional RobertsonWalker space-time, when the source for energy momentum tensor is bulk viscous fluid. Section III is devoted to the solutions of the field equations under some physical conditions. In Section IV, we discuss some physical and kinematical properties of the cosmological model and Section V contains some conclusions.

\section{II.FIELD EQUATIONS}

We consider $(2+1)$ dimensional Robertson-Walker line element [32]

$$
d s^{2}=d t^{2}-R^{2}(t)\left(d r^{2}+r^{2} d \theta^{2}\right),
$$

where the spatial curvature is taken to be zero and the energy momentum tensor $T_{i j}$ for a viscous fluid is given by

With

$$
T_{i j}=(\rho+\bar{p}) u_{i} u_{j}-\bar{p} g_{i j}
$$

$$
\bar{p}=p-\zeta \theta
$$

where $\zeta$ is the bulk viscosity coefficients, $\theta$ is scalar expansion, $\rho$ and $p$ are the energy density and isotropic pressure respectively.

Using commoving coordinate system $u^{i}=\delta_{0}^{i}$ satisfying $g_{i j} u^{i} u_{j}=1$, the Einstein field equations

$$
R_{i j}-\frac{1}{2} R g_{i j}=T_{i j}(i, j=0,1,2)
$$

for the metric (4) with energy momentum tensor (5) are given

$$
\begin{gathered}
\frac{\dot{R}^{2}}{R^{2}}=\rho \\
\frac{2 \ddot{R}}{R}=-p+2 \zeta\left(\frac{\dot{\mathrm{R}}}{\mathrm{R}}\right)
\end{gathered}
$$

Here, dot $(\cdot)$ denotes differentiation with respect to time $t$ only and expansion scalar $\theta$ is given by

$$
\theta=u_{; i}^{i}=2 \frac{\dot{\mathrm{R}}}{\mathrm{R}}
$$

\section{SOLUTIONS OF THE FIELD EQUATIONS}

There are two field equations (8) and (9) with four unknowns namely one scale factor $R$ and three physical quantities $\rho, p$ and $\zeta$. Therefore to obtain an exact solution of the field equations, we need two more relations connecting these variables. Hence we use the following plausible physical conditions.

i) Variation of Hubble's parameter proposed by Berman [33] that yields a constant deceleration parameter models of the universe which is defined as

$$
q=-\frac{R \ddot{R}}{\dot{R}^{2}}=\text { constant }
$$

ii) For a barotropic fluid the combine effects of the proper pressure and the bulk viscous pressure can be expressed as

$$
\bar{p}=p-2 \zeta H=\varepsilon \rho, \quad p=\varepsilon_{0}(11)
$$

where $\varepsilon=\varepsilon_{0}-\alpha \quad\left(0 \leq \varepsilon_{0} \leq 1\right)$ and $\varepsilon, \varepsilon_{0}$ and $\alpha$ are constants.

Now, the Eqn. (10) admits the solution

$$
R=(a t+b)^{1 / q+1},
$$

where $a$ and $b$ are constants of integration. Eq. (12) implies that the condition for expansion of the universe is $q+1>$ 0 .

With suitable choice of coordinates and constants i.e. taking $a=1$ and $b=0$, the metric (4) can be written as

$$
d s^{2}=d t^{2}-t^{2 / q+1}\left(d r^{2}+r^{2} d \theta^{2}\right)
$$

Eq. (13) represents the viscous fluid cosmological model in $(2+1)$ dimensional gravity theory. 


\section{PHYSICAL AND KINEMATICAL PROPERTIES AND DISCUSSION}

The physical and kinematical quantities for the model (13) have the following expressions.

The scale factor $R$ is given by

$$
R=t^{1 / q+1}
$$

Spatial volume: $V_{2}=R^{2}=t^{2 / q+1}$

Expansion scalar: $\theta=u_{; i}^{i}=2 \frac{\dot{\mathrm{R}}}{\mathrm{R}}=\frac{2}{(q+1) t}$

Hubble's parameter:

$H=\frac{1}{2}\left(H_{1}+H_{2}\right)=\frac{1}{2}\left(\frac{\dot{R}}{R}+\frac{\dot{R}}{R}\right)=\frac{\dot{R}}{R}=\frac{1}{(q+1) t}$

Energy density: $\rho=\left(\frac{\dot{R}}{R}\right)^{2}=\frac{1}{[(q+1) t]^{2}}$

Isotropic pressure: $p=\varepsilon_{0} \rho=\frac{\varepsilon_{0}}{[(q+1) t]^{2}}$

Coefficient of bulk viscosity:

$$
\zeta=\frac{1}{2 H}\left[\left(\varepsilon-\varepsilon_{0}\right) \rho\right]=\frac{\varepsilon-\varepsilon_{0}}{2(q+1) t}
$$

Shear scalar: $\sigma^{2}=\frac{1}{2}\left[\sum_{i} H_{i}^{2}-\frac{1}{2} \theta^{2}\right]=0$

Anisotropic parameter:

$$
A_{m}=\frac{1}{2} \sum_{i}\left(\frac{H_{i}-H}{H}\right)^{2}=0
$$

Using the above results, we now discuss the behavior of the cosmological model (13).

The result (15) shows that the model is expanding with time since $q+1>0$. It can be observed that the model given by (13) has no initial singularity at $t=0$. It can also be observed that, the Hubble parameter $H$, expansion scalar $\theta$, matter density $\rho$ and pressure $p$ decreases with time and approach zero as $t \rightarrow \infty$ and all diverges at $t=0$. Also shear scalar $\sigma$ and anisotropic parameter $A_{m}$ vanishes, which indicates that shape of the universe remains unchanged during the evolution and universe becomes isotropic and shear free.

\section{V.CONCLUSION}

Bulk viscosity in $(2+1)$ dimensional space plays a significant role in the discussion of early stages of the evolution of universe and in inflationary cosmology. Hence we have investigated here bulk viscosity cosmological model in the frame work of $(2+1)$ dimensional space-time. The model is obtained using the special law of variation for Hubble's parameter proposed by Berman [33] and the bar tropic equation of state for pressure and energy density. It is observed that the model is expanding, nonsingular and nonrotating. It is also observed that all the physical and kinematical parameters of the model diverges when $t=0$ and vanish when $t$ is infinitely large. Also we have $\frac{\sigma^{2}}{\theta^{2}}=0$ and anisotropic parameter $A_{m}=0$, which indicate that the model is not anisotropic in nature and remains isotropic throughout the evolution. The bulk viscosity in the model decreases with time leading to inflationary model.

\section{REFERENCES}

[1] S. Deser, R. Jackiw and G. 't Hooft, Ann. Phys. 152, 220, (1984).

[2] G. Clement, Int. J. Theor. Phys. A: Math. Gen. 14, 2353, (1981).

[3] S. Deser and P.O. Mazur, Class. Quantum Grav. 2, L51,(1985).

[4] M. A. Melvin, Class Quantum Grav. 3, 117 (1986)

[5] J. R. Gott, J. Z. Simon and M. Alpert, Gen. Rel. Grav.[6], 1019, (1986).

[7] M. Rocek and R. Williams, Class. Quantum Grav. 2, 701, (1985).

[8] S. Giddings, J. Abbott and K. Kuchar, Gen. Rel. Grav. 16, 751,(1984).

[9] J. R. Gott and M. Alpert, Gen. Rel. Grav. 16, 243, (1984).

[10] J. D. Barrow, A. B. Burd and D. Lancaster, Class. Quantum Grav. 3 , 551, (1986).

[11] S. Deser and B. Laurent, Gen. Rel. Grav. 18, 617(1986).

[12] S. Deser, Class. Quantum Grav. 1, L1(1984).

[13] Edward Witten, Nuclear Physics, B 311, $46-78$ (1988/89).

[14] N.J. Cornish and N.E. Frankel, Physical Review D, Volume 43, Number 8, 2555-2565(1991).

[15] Bin Wang and Elcio Abdalla, Physics Letters B 466,122126(1999).

[16] Ranjan Sharma et.al, Ranjan Sharma, Shyam Das, Farook Rahaman and Gopal Chandra Shit, Astrophys Space Sci. 359:40 (2015).

[17] Yun He and Meng-Sen Ma, Physics Letters B, 774, 229234.(2017)

[18] D. R. K. Reddy, R. Santhi Kumar, and T. V. Pradeep Kumar, "Kaluza-Klein universe with cosmic strings and bulk viscosity in a scalar-tensor theory of gravitation," International Journal of TheoreticalPhysics, vol. 52, no. 4, pp. 1214-1220, (2013)

[19] D. S'aez and V. J. Ballester, "A simple coupling with cosmological implications," Physics Letters A, vol. 113, no. 9, pp. 467-470, (1986).

[20] R. L. Naidu, K. Dasu Naidu, K. Shobhan Babu, and D. R. K. Reddy, "A five dimensional Kaluza- Klein bulk viscous string cosmological model in Brans-Dicke scalar- tensor theory of gravitation," Astrophysics and Space Science, vol. 347, no. 1, pp.197-201,(2013).

[21] C. Brans and R. H. Dicke, "Mach's principle and a relativistic theory of gravitation," Physical Review, vol.124, no. 3, pp. 925 935, (1961).

[22] D. R. K. Reddy, R. L. Naidu, K. Dasu Naidu, and T. RamPrasad, "LRS Bianchi type-II universe with cosmic strings and bulk viscosity in a modified theory of gravity," Astrophysics and Space Science, vol. 346, no. 1, pp. 219-223, (2013).

[23] T. Harko, F. S. N. Lobo, S. Nojiri, and S. D. Odintsov, " $f(R, T)$ gravity," Physical Review D, vol. 84, Article ID 024020, 11 pages, (2011).

[24] D. R. K. Reddy, R. L. Naidu, K. Dasu Naidu, and T. Ram Prasad, "Kaluza-Klein universe with cosmic strings and bulk viscosity in $f(R, T)$ gravity," Astrophysics and Space Science, vol. 346, no.1, pp. 261-265, (2013).

[25] R. L. Naidu, D. R. K. Reddy, T. Ramprasad, and K.V. Ramana, "Bianchi type-V bulk viscous string cosmological model in $f$ $(R, T)$ gravity," 
Astrophysics and Space Science, (2013).

[26] D. R. K. Reddy, R. L. Naidu, T. Ramprasd, and K. V. Ramana, "LRS Bianchi type-II bulk viscous cosmic string model in a scale covariant theory of gravitation," Astrophysics and Space Science,(2013).

[27] V. Canuto, S. H. Hsieh, andP. J.Adams, "Scale-Covariant theory of gravitation and astrophysical applications," Physical Review Letters, vol. 39, no. 8, pp. 429-432, (1977).

[28] D. R. K. Reddy, Ch. Purnachandra Rao, T. Vidyasagar, and R. Bhuvana Vijaya, Advances in High Energy Physics, Volume, Article ID 609807, 5 pages (2013).

[29] D. S'aez and V. J. Ballester, "A simple coupling with cosmological implications," Physics Letters A, vol. 113, no. 9, pp. 467-470, (1986).

[30] Shri Ram_, Priyanka Kumari† Cent. Eur. J. Phys.,12(10), 744754,DOI: 10.2478/s11534-014-0494-3(2014).

[31] Mishra R K and Chand A Phys Astron Int J. 1(5): 00031,(2017).

[32] Brans C, Dicke RH) Mach's Principle and a Relativistic Theory of Gravitation. Physical Review journal Archive 124(3), (1961).

[33] N.J. Cornish, N.E. Frankel, Phys. Rev. D 43. 2555, (1991).

[34] M. S. Berman, "A special law of variation for Hubble's parameter,"Nuvo Cimento B, vol. 74, no. 2, pp. 182-186, (1983).

\section{AUTHORS PROFILE}

Mr. G R Avachar pursed M. Sc. (Mathematics) and Ph.D. in 1986 and 2007. He is currently working as Associate Professor in Department of Mathematics, S. S. E. S. Amravati's Science College, Nagpur. He was Chairman of Board of Studies of Mathematics during 2005-2010 and presently member of Board of Studies in R.T.M. Nagpur University, Nagpur during 2005-2010. He has published more than 10 research papers in reputed international journals (SCI \& Web of Science)/conferences His main research work focuses on Theory of Relativity. He has 30 years of teaching experience and 10 years of research. 